\title{
Best Practices for Managing Turnover in Data Science Groups, Teams, and Labs
}

\section{A Report from the Berkeley Institute for Data Science's Best Practices in Data Science Series}

${ }^{*}$ Corresponding author: dsholler@berkeley.edu

${ }^{\dagger}$ These authors contributed equally to this work, order is alphabetical

¥These authors contributed equally to this work, order is alphabetical

Published: 05 Mar 2019

DOI: 10.31235/osf.io/wsxru

License: Creative Commons Attribution (CC BY 4.0 Intl)

\section{Dan Sholler ${ }^{1,2{ }^{*}}$, Diya Das ${ }^{1,3 \dagger}$, Fernando Hoces de la Guardiaa ${ }^{4 \dagger}$, Chris Hoffman $^{5 \ddagger}$, François Lanusse ${ }^{1,6,7 \ddagger}$, Nelle Varoquaux ${ }^{1,11 \ddagger}$, Rolando Garcia $^{8 \ddagger}$, R. Stuart Geiger ${ }^{1 \ddagger}$, Shana McDevitt ${ }^{9 \ddagger}$, Scott Peterson ${ }^{10 \ddagger}$, Sara Stoudt ${ }^{1,11 \neq}$}

${ }^{1}$ Berkeley Institute for Data Science, University of California, Berkeley

2 rOpenSci

3 Department of Molecular and Cell Biology, University of California, Berkeley

${ }^{4}$ Berkeley Initiative for Transparency in the Social Sciences, University of California, Berkeley

${ }^{5}$ Research IT, University of California, Berkeley

${ }^{6}$ Berkeley Center for Cosmological Physics, University of California, Berkeley

7 Foundations of Data Analysis Institute, University of California, Berkeley

8 RISELab, University of California, Berkeley

${ }^{9}$ QB3 Vincent J. Coates Genomics Sequencing Laboratory, University of California, Berkeley

10 Morrison Library and Graduate Services Library, University of California, Berkeley

${ }^{11}$ Department of Statistics, University of California, Berkeley

\section{Abstract:}

Turnover is a fact of life for any project, and academic research teams can face particularly high levels of people who come and go through the duration of a project. In this article, we discuss the challenges of turnover and some potential practices for helping manage it, particularly for computational- and data-intensive research teams and projects. The topics we discuss include establishing and implementing data management plans, file and format standardization, workflow and process documentation, clear team roles, and check-in and check-out procedures.

Recommended citation: Dan Sholler, Diya Das, Fernando Hoces de la Guardia, Chris Hoffmann, François Lanusse, Nelle Varoquaux, Rolando Garcia, R. Stuart Geiger, Shana McDevitt, Scott Peterson, Sara Stoudt. “Best Practices for Managing Turnover in Data Science Groups, Teams, and Labs." BIDS Best Practices in Data Science Series. Berkeley, CA: Berkeley Institute for Data Science. 2019. doi:10.31235/osf.io/wsxru 


\section{Introduction}

Turnover, or the arrival and departure of personnel from a project, is common in academic research teams. Students, postdocs, research staff, visiting scholars, and collaborators come and go from projects as their career and research opportunities change. For this reason, labs and teams should have mechanisms in place to ensure smooth transitions from one team member to the next. Data-intensive research teams are no different; in fact, the nature of data-intensive research makes turnover management essential to sustaining research efforts. As we have discussed in previous Best Practices blog posts and meetings, data intensive research teams often are heterogeneous in members' backgrounds and expertise, require collaboration across disciplinary and subdisciplinary boundaries, and weave together smaller, easier to manage projects into larger, more complex research systems.

In this post, we discuss some of the challenges presented by turnover and lay out some best (or "good enough") practices for contending with the challenges. First, we explain how data collection and accessioning can be standardized by first piloting the collection process and documenting it in sufficient detail. Next, we turn to issues around funding requirements and turnover, arguing that teams should know and consider funding agency requirements for data-intensive research (e.g., accessing, using, and publishing datasets) and document team member roles for managing adherence to funder rules. We conclude by emphasizing check-in and check-out procedures as a foundational way of managing turnover in data-intensive research teams and acknowledge the numerous questions unaddressed in our working group meeting.

Best practices for data-intensive research have been the subject of several papers, notably a paper of the same name and a follow-up paper by Greg Wilson and colleagues (Wilson et al., 2014, 2017). We encourage all research groups to read and reflect on how those practices may be of use. Our group's discussion touched on several of the same areas, though there is incomplete overlap as the focus of our discussion was on how the difficulties posed by regular personnel turnover can be mitigated by project pre-planning and documentation.

\section{Data management should be a part of the project plan}

Our Best Practices series meetings are centered on developing generalizable practices to promote reproducible data-intensive research. The components of reproducibility include the "the public availability of the data and software" (Rokem et al., 2018 , p. 7). Best practices for creating reproducible scripts and software packages 
is a common topic of discussion-researchers who conduct data-intensive research are often familiar with Git and GitHub or other software development and version control systems, recognize the importance of code documentation, and engage with one another in robust open source software communities.

Less often discussed are issues around data management, such as data accessioning and permanent storage, dataset sharing, and data citation. Yet emergent data services offer several ways forward in developing sound data management practices. Examples include the Center for Open Science's Open Science Framework, the newly-formed partnership between California Digital Library's DASH and Data Dryad (Simms, 2018), discipline-specific repositories such as GenBank, and multipurpose services such as figshare ${ }^{2}$ or Zenodo. ${ }^{3}$ DataVerse ${ }^{4}$ is a useful resource for evaluating and comparing data management tools and services for your project.

\section{Data management plans/protocols (DMPs)}

Armed with knowledge of the available tools and services, research teams can begin to develop data management plans (DMP) and consider how to address turnover within the plan. Although DMPs are now a common (and sometimes frustrating) mandate from many funders, teams often do not recognize the beneficial role they can play in managing turnover. For example, in our discussion, we emphasized that DMPs should document the roles of each person in accessing, storing, backing up, locating, and otherwise stewarding the data.

Commonly, much of this data management work is handled by "temporary" employees such as students and postdocs; in turn, documentation of the processes and responsibilities is paramount to the success of the project. Additionally, one or more team members should maintain responsibility for adhering to funder, journal, and institutional policies about data access, storage, and sharing. This member can consult with your institution's Research Data Management (RDM) service, where available (for example, UC-Berkeley's RDM offers consulting ${ }^{5}$ ). The California Digital Library's DMPTool ${ }^{6}$ is also a robust tool for creating a tailored, team-specific DMP (see also Sallans and Donnelly 2012 and Starr et al. 2012).

We noted that for individual research projects, though the protocols are often docu-

\footnotetext{
1 https://www.ncbi.nlm.nih.gov/genbank/

2 https://figshare.com

3 https://zenodo.org

4 https://dataverse.org

${ }^{5}$ See more information at https://researchdata.berkeley.edu

6 https://dmptool.org
} 
mented for pre-approval, recording of experimental observations or intermediate data outputs is not necessarily standardized. Furthermore, data management can vary by data type; it is important to construct a plan that aligns with your pipeline and is accessible and easy to use for your research team, no matter members' degree of technical proficiency (Geiger et al., 2018).

\section{Standardization and documentation of data and file formats}

With multiple individuals involved, it is especially important to not just plan for standardization, but also document standards as part of a project plan at the start of a project. We observed that journal and funding agency requirements and infrastructure can have a large effect on degree of standardization. In genomics, for example, raw sequence data is often expected to be shared at time of publication, and the National Cancer Biotechnology Institute maintains a Sequence Read Archive for data deposition ${ }^{7}$ (see also Leinonen et al. 2010).

We also noted that large research consortia, by their nature, must set standards for data collection types. The Human Genome Project, for example, appears to be responsible for the development of several file formats (Kent et alı, 2002). Finally, it is important to acknowledge that software availability has shaped the development and adoption of other file formats (Cock et al., 2009). Within a research group, standardization of file formats and file organization is an important for the development of reproducible analysis pipelines.

Setting internal standards around file structure and file management is one of the practices for reproducibility that can provide the biggest bang for a (very small) buck. Project TIER ${ }^{8}$ is an excellent resource as it provides detailed templates and clear specifications on how, when, and where documentation should be created (see also Medeiros and Ball 2017). In addition, workflow diagrams can be of assistance in helping team members understand and adhere to project standards for data storage and management. Some good examples of data workflow diagrams can also be found in the edited collection The Practice of Reproducible Research (Kitzes et al., 2018).

\section{Documenting data collection and analysis procedures}

While DMPs are more standardized in the physical and life sciences, those of us from the social sciences (like economics, political science and psychology) also saw

7 https://www.ncbi.nlm.nih.gov/sra

8 https://www.projecttier.org/ 
potential in adopting some of the protocols and best practices outlined above. There is plenty of space for learning, here we suggest just two examples. First, the concept of a scientific lab notebook could be adopted in social science survey data collection to create a log of all the main methodological decisions (designing the instruments, field and data intake logistics, etc.; see Schreier et al. 2006 ). Currently many research groups track those decisions in an ad-hoc manner, but a standardized procedure would help with later publication of all the materials.

Second, the use of data from administrative records (e.g., tax, hospital, or insurer records) is an ever-growing practice in social science. One key problem is that until now, most of the access to these rich data sets is restricted to a very small number of researchers with the right connections. The absence of a clear pipeline to access administrative records is justified on the the protection of confidentiality and privately identifiable information. We think that this debate could be enriched by the experiences in genomics and other data-intensive disciplines that must deal with the trade-off of obtaining access to highly sensitive information and sharing data to ensure reproducibility. Unfortunately, we did not have time for a full discussion during this meeting.

DMPs can be difficult to construct without empirical grounding and contextual expertise (i.e., without having begun data collection in the field). Piloting can aid in the process of constructing a plan. In the pilot phase, team members can begin delineating each member's roles and responsibilities. Even when a DMP exists, piloting can help to reevaluate the feasibility of the proposal. Additionally, it might also be useful to discuss and document authorship and credit attribution during this process (i.e., which roles and responsibilities correspond to authorship opportunities and orders).

\section{Develop, Document, and Implement Check-In and Check- Out Procedures}

While data standardization and documentation was a significant focus of our discussion, the larger question of adaptability to turnover remained. From our experience, we have found that implementation of check-in and check-out procedures can help. While such procedures are often found in many groups from a regulatory perspective, we suggest extending them to address project-specific concerns. At check-in, we noted that it was important to discuss macro-scale questions in project organization, addressing both technical requirements and the project management scheme. 
We also found it helpful to discuss desired outcomes and goalposts, both for the project and for individual contributors, to ensure that contributors have a stake in project success. Finally, we found it helpful to discuss all aspects of "how the team works" - while many modes of work might be acceptable for any given project, reducing friction with existing workflows is beneficial. These topics ranged from file structures and coding styles to codes of conduct and managing interpersonal interactions.

Ideally, much of project documentation would be written during the course of a project, but sometimes best intentions escape us. In addition, a team member's tacit knowledge still requires documentation. At the departure of a research group member, we recommend a checkout procedure for computational projects alongside any existing checkout for administrative or experimental purposes. We also recommend a review of job responsibilities, project, data and backup organization against the documentation, in the event that items were added or removed without prior documentation, so that this knowledge can be passed on to the next person.

We also explicitly recommend a review of any services that are tied to institutional email addresses, as they may become deactivated. Access to resources and data should be reviewed in this context as well, to ensure not only that any restrictions on access after departure are met, but also that remaining team members have rights to manage access. From an administrative standpoint, we also recommend collecting non-institutional contact information from the departing team member, should further questions arise, or for the sake of future publications.

Due to the brevity of our discussion, numerous topics were not addressed. Examples include a more in-depth discussion of gaining and managing access to sensitive data, and administrative concerns (e.g., formalizing collaborations) (Playford et al., 2016). Like all Best Practices lunches, this discussion raised many topics of discussion for future meetings. We continue to recommend that researchers adopt this format for discussions in their own communities, so they are able to address the nuances of their particular situations. However, we also derived great benefit from an interdisciplinary meeting that allowed us to contrast the practices of different fields.

\section{Acknowledgments}

This work was funded by the Gordon \& Betty Moore Foundation (Grant GBMF3834) and Alfred P. Sloan Foundation (Grant 2013-10-27), as part of the Moore-Sloan Data Science Environments grant to the University of California, Berkeley. 


\section{References}

Cock PJ, Fields CJ, Goto N, Heuer ML, Rice PM. The Sanger FASTQ file format for sequences with quality scores, and the Solexa/Illumina FASTQ variants. Nucleic acids research. 2009; 38(6):1767-1771. https://www.ncbi.nlm.nih.gov/pmc/articles/PMC2847217/.

Geiger RS, Sholler D, Culich A, Martinez C, de la Guardia FH, Lanusse F, Ottoboni K, Stuart M, Vareth M, Varoquaux N, Stoudt S, van der Walt S. Challenges of Doing Data-Intensive Research in Teams, Labs, and Groups. In: BIDS Best Practices in Data Science Series. Berkeley, CA: Berkeley Institute for Data Science; 2018.

https://doi.org/10.31235/osf.io/a7b3m.

Kent WJ, Sugnet CW, Furey TS, Roskin KM, Pringle TH, Zahler AM, Haussler D. The human genome browser at UCSC. Genome research. 2002; 12(6):996-1006.

https://genome.cshlp.org/content/12/6/996.full.

Kitzes J, Turek D, Deniz F. The Practice of Reproducible Research : Case Studies and Lessons from the Data-Intensive Sciences. Oakland: University of California Press; 2018. http://practicereproducibleresearch.org.

Leinonen R, Sugawara H, Shumway M, Collaboration INSD. The Sequence Read Archive. Nucleic Acids Research. 2010; 39. https://www.ncbi.nlm.nih.gov/pubmed/21062823.

Medeiros N, Ball RJ. Teaching Integrity in Empirical Economics: The Pedagogy of Reproducible Science in Undergraduate Education. In: Hensley MK, Davis-Kahl S, editors. Undergraduate Research and the Academic Librarian: Case Studies and Best Practices Chicago: Association of College \& Research Libraries; 2017.

https://scholarship.haverford.edu/cgi/viewcontent.cgi?article=1189.

Playford CJ, Gayle V, Connelly R, Gray AJ. Administrative social science data: The challenge of reproducible research. Big Data \& Society. 2016; 3(2):2053951716684143. https://doi.org/10.1177/2053951716684143, doi: 10.1177/2053951716684143.

Rokem A, Marwick B, Staneva V. Assessing Reproducibility. In: Kitzes J, Turek D, Deniz F, editors. The Practice of Reproducible Research : Case Studies and Lessons from the Data-Intensive Sciences Oakland: University of California Press; 2018. https://www.practicereproducibleresearch.org/core-chapters/2-assessment.html.

Sallans A, Donnelly M. DMP Online and DMPTool: Different Strategies Towards a Shared Goal. International Journal of Digital Curation. 2012; 7(2):123-129. https://doi.org/10.2218/ijdc.v7i2.235.

Schreier AA, Wilson K, Resnik D. Academic research record-keeping: Best practices for individuals, group leaders, and institutions. Academic medicine: journal of the Association of American Medical Colleges. 2006; 81(1):42. https://www.ncbi.nlm.nih.gov/pmc/articles/PMC3943904/. 
Simms S, Letter to the Community: CDL and Dryad Partnership. California Digital Library; 2018. https://www.cdlib.org/cdlinfo/2018/05/30/

letter-to-the-community-cdl-and-dryad-partnership/.

Starr J, Willett P, Federer L, Horning C, Bergstrom ML. A Collaborative Framework for Data Management Services: The Experience of the University of California. Journal of eScience Librarianship. 2012; 1(2). https://escholarship.umassmed.edu/jeslib/vol1/iss2/7, doi: 10.7191/jeslib.2012.1014.

Wilson G, Aruliah DA, Brown CT, Chue Hong NP, Davis M, Guy RT, Haddock SHD, Huff KD, Mitchell IM, Plumbley MD, Waugh B, White EP, Wilson P. Best Practices for Scientific Computing. PLoS Biology. 2014 Jan; 12(1):e1001745. doi: 10.1371/journal.pbio.1001745.

Wilson G, Bryan J, Cranston K, Kitzes J, Nederbragt L, Teal TK. Good enough practices in scientific computing. PLOS Computational Biology. 2017 Jun; 13(6):e1005510. doi: 10.1371/journal.pcbi.1005510. 